\title{
Association between wetland presence and incidence of Salmonella enterica serotype Javiana infections in selected US sites, 2005-2011
}

\author{
J. Y. HUANG ${ }^{1}$, M. E. PATRICK ${ }^{1}$, J. MANNERS $^{2}$, A. R. SAPKOTA ${ }^{3}$, \\ K. J. SCHERZINGER ${ }^{4}$, M. TOBIN-D'ANGELO ${ }^{5}$, O. L. HENAO ${ }^{1}$, D. J. COLE ${ }^{1}$ \\ AND A. R. VIEIRA ${ }^{1}$ \\ ${ }^{1}$ Centers for Disease Control and Prevention, Atlanta, Georgia, USA \\ ${ }^{2}$ Tennessee Department of Health, Nashville, Tennessee, USA \\ ${ }^{3}$ University of Maryland, College Park, Baltimore, Maryland, USA \\ ${ }^{4}$ New Mexico Emerging Infections Program, University of New Mexico, Albuquerque, New Mexico, USA \\ ${ }^{5}$ Georgia Department of Public Health, Atlanta, Georgia, USA
}

Received 30 March 2017; Final revision 3 July 2017; Accepted 17 July 2017;

first published online 14 August 2017

\section{SUMMARY}

Salmonella causes an estimated 1.2 million illnesses annually in the USA. Salmonella enterica serotype Javiana (serotype Javiana) is the fourth most common serotype isolated from humans, with the majority of illnesses occurring in southeastern states. The percentage of wetland cover by wetland type and the average incidence rates of serotype Javiana infection in selected counties of the Foodborne Disease Active Surveillance Network (FoodNet) were examined. This analysis explored the relationship between wetland environments and incidence in order to assess whether regional differences in environmental habitats may be associated with observed variations in incidence. Findings suggest that environmental habitats may support reservoirs or contribute to the persistence of serotype Javiana, and may frequently contribute to the transmission of infection compared with other Salmonella serotypes.

Key words: Incidence, Salmonella serotype Javiana, wetlands.

\section{INTRODUCTION}

Salmonella causes an estimated $1 \cdot 2$ million illnesses annually in the United States [1]. Salmonella enterica serotype Javiana (serotype Javiana) is the fourth most common serotype isolated from humans, with the majority of illnesses occurring in southeastern states [2]. Since the late 1990s, the number of reported serotype Javiana infections has been increasing. During 2001-2011, serotype Javiana increased by $175 \%$, the largest increase among the top 20

\footnotetext{
* Author for correspondence: J. Y. Huang, Centers for Disease Control, Enteric Disease Epidemiology Branch-FoodNet, 1600 Clifton Rd NE, MS C-09, Atlanta, GA 30033, USA.

(Email: uzo0@cdc.gov)
}

Salmonella serotypes [2]. Risk factors that have been associated with serotype Javiana infection through outbreak investigations and sporadic case-control studies include consumption of contaminated produce (e.g., tomatoes, sprouts, and lettuce), well water, and contact with animals (e.g., reptiles and amphibians) [3-5]. These findings suggest that the common meat and poultry reservoirs of other Salmonella serotypes are less associated with infection caused by serotype Javiana. Salmonella can also persist in aquatic environments and different types of wetland are suspected to host environmental and animal reservoirs of serotype Javiana [6]. To better understand possible sources of serotype Javiana infections, the percentage of wetland covered and the average incidence rates of serotype Javiana infection in selected counties of the 
Foodborne Disease Active Surveillance Network (FoodNet) were examined.

\section{METHODS}

FoodNet is the principal foodborne disease surveillance component of the Centers for Disease Control and Prevention (CDC)'s Emerging Infections Program, a collaboration among CDC, the US Department of Agriculture's Food Safety and Inspection Services (USDA-FSIS), the Food and Drug Administration (FDA), and ten state health departments. FoodNet sites include the states of Connecticut, Georgia, Maryland, Minnesota, New Mexico, Oregon, and Tennessee, and selected counties in California, Colorado, and New York. FoodNet staff conduct active population-based surveillance for culture-confirmed cases of bacterial infections, including Salmonella, meaning all cases within the catchment area are ascertained. Based on results from previous studies, four FoodNet sites (Georgia, Maryland, New Mexico, and Tennessee) with the highest incidence of serotype Javiana infections (at least 1.0 case per 100000 population annually) were selected during 2005-2011 study period. The total number of serotype Javiana cases was aggregated by 311 FoodNet counties. The smallest level of geographic unit used was county. Individual addresses of cases were not available for geocoding. The year and county-specific incidence rates and average annual incidence rates (AvIR) were calculated. The intercensal estimates from US census data for 2005 to 2011 were used as denominators, and AvIRs were mapped by county in each state using ArcGIS [7].

The data on wetlands were extracted from the US Fish and Wildlife Services (US-FWS)'s National Wetland Inventory (US-FWS 2012). US-FWS classifies wetland into five broad categories: marine (oceans), estuarine (tidal and salt water marshes), riverine (rivers and streams), lacustrine (lakes and their fringe areas), and palustrine (non-tidal, inland wetland, such as swamps, bogs, and fens). Palustrine is further divided into freshwater forested/scrubshrub, freshwater emergent, and freshwater pond. Freshwater forested/scrub-shrub consists of forested swamps. In contrast, freshwater emergent consists of herbaceous marsh, fend, wale, wet meadow, and seasonally wet areas that support hydric soils and hydrophitic vegetation [8]. A separate map layer of each of the following distinct types of wetland was created using ArcGIS: freshwater forested/scrub-shrub, freshwater emergent, freshwater pond, riverine, and lakes. Marine and estuarine wetlands were excluded because they were not present in the counties included in the study. Using state specific data, the percentage of each county covered by the different types of wetland (wetland percent coverage) was calculated. A Spearman's rank correlation coefficient was used to assess the correlation between the AvIR of serotype Javiana and percentage of coverage by wetland type. Fisher transformation was used to evaluate the significance of the correlation coefficients, $95 \%$ confidence interval with a $P$-values $<0.05$ was considered to be statistically significant [9]. Strength of the correlation coefficient was defined to be very weak $(0 \cdot 00-0 \cdot 19)$, weak $(0 \cdot 2-0 \cdot 39)$, moderate $(0 \cdot 40-0 \cdot 59)$, strong $(0 \cdot 60$ $0 \cdot 79)$, or very strong $(0 \cdot 8-1 \cdot 0)$. State-specific maps were created to visualize the percentage of the county covered by each wetland type as well as county AvIR.

Salmonella enterica serotype Enteritidis (serotype Enteritidis) cases were ascertained during the same time period, and correlation coefficients between wetland type and incidence of serotype Enteritidis were calculated. serotype Enteritidis was used as a referent serotype because it is the most common serotype detected by FoodNet, and is typically associated with foodborne rather than environmental transmission [10], which is why a correlation was not expected between wetland coverage and the incidence of serotype Enteritidis. The correlation coefficients for serotype Javiana were compared with correlation coefficients for serotype Enteritidis. All of the data analyses were conducted using SAS 9.3 [11].

\section{RESULTS}

The wetland percent coverage for each county ranged from $0 \cdot 03 \%$ to $90 \cdot 1 \%$, with fresh forested/scrub-shrub being the most common wetland type in all four states. Average wetland percent coverage for each type of wetland is shown in Table 1. Counties in Georgia had the highest wetland percent coverage for all wetland types except riverine wetland. Tennessee counties had the highest percent coverage for riverine wetland. Counties in New Mexico had less than $0 \cdot 1 \%$ wetland percent coverage for the four types of wetland. Figure 1 shows the geographic variation of wetland percent coverage with fresh forested/ scrub-shrub wetland and serotype Javiana AvIR by county.

There were 2542 serotype Javiana cases reported in the four states during 2005-2011 (Table 1). Georgia 
Table 1. Average percentage of county covered by specific wetland and average incidence rate of Salmonella enterica serotype Javiana and Salmonella enterica serotype Entertidis, by county in Georgia, Maryland, New Mexico,

Tennessee, 2005-2011

\begin{tabular}{|c|c|c|c|c|}
\hline State & Georgia & Maryland & New Mexico & Tennessee \\
\hline Total number of counties & 159 & 24 & 33 & 95 \\
\hline $\begin{array}{l}\text { Average percentage of county covered by } \\
\text { types of wetland }\end{array}$ & $\%$ (range) & $\%$ (range) & $\%$ (range) & $\%$ (range) \\
\hline Freshwater emergent & $0.58(0 \cdot 01-3.99)$ & $0 \cdot 41(0 \cdot 02-1 \cdot 84)$ & $0 \cdot 07(0 \cdot 00-0 \cdot 33)$ & $0 \cdot 22(0 \cdot 30-3 \cdot 28)$ \\
\hline Freshwater forested/shrub & $12 \cdot 00(0 \cdot 02-90 \cdot 05)$ & $4 \cdot 88(0 \cdot 06-22 \cdot 01)$ & $0 \cdot 06(0 \cdot 00-0 \cdot 29)$ & $3 \cdot 53(0 \cdot 01-69 \cdot 42)$ \\
\hline Freshwater pond & $0 \cdot 59(0 \cdot 05-2 \cdot 46)$ & $0 \cdot 22(0 \cdot 02-1 \cdot 84)$ & $0 \cdot 03(0 \cdot 00-0 \cdot 13)$ & $0 \cdot 31(0 \cdot 03-0 \cdot 94)$ \\
\hline Lake & $6 \cdot 00(0 \cdot 01-98 \cdot 08)$ & $0 \cdot 54(0 \cdot 00-3 \cdot 31)$ & $0 \cdot 00$ & $12 \cdot 38(0 \cdot 01-84 \cdot 09)$ \\
\hline Riverine & $1 \cdot 11(0 \cdot 00-7 \cdot 21)$ & $1 \cdot 19(0 \cdot 04-5 \cdot 30)$ & $0 \cdot 00(0 \cdot 00-0 \cdot 07)$ & $5 \cdot 28(0 \cdot 98-98 \cdot 26)$ \\
\hline \multicolumn{5}{|l|}{ Salmonella serotype Javiana } \\
\hline Total number of cases & 1911 & 180 & 170 & 285 \\
\hline Mean AvIR ${ }^{\mathrm{a}}$ (range) & $5 \cdot 58(0 \cdot 00-40 \cdot 4)$ & $1 \cdot 35(0 \cdot 00-9 \cdot 78)$ & $1 \cdot 07(0 \cdot 00-10 \cdot 0)$ & $0 \cdot 8(0 \cdot 00-5 \cdot 90)$ \\
\hline \multicolumn{5}{|l|}{ Salmonella serotype enteritidis } \\
\hline Total number of cases & 1173 & 1598 & 143 & 622 \\
\hline Mean AvIR ${ }^{\mathrm{a}}$ (range) & $2 \cdot 17(0 \cdot 00-14 \cdot 3)$ & $4 \cdot 0(1 \cdot 40-18 \cdot 90)$ & $1 \cdot 43(0 \cdot 00-7 \cdot 0)$ & $1 \cdot 64(0 \cdot 00-9 \cdot 90)$ \\
\hline
\end{tabular}

Abbreviation: AvIR, average incidence.

${ }^{a}$ Average incidence by county per 100000 person.

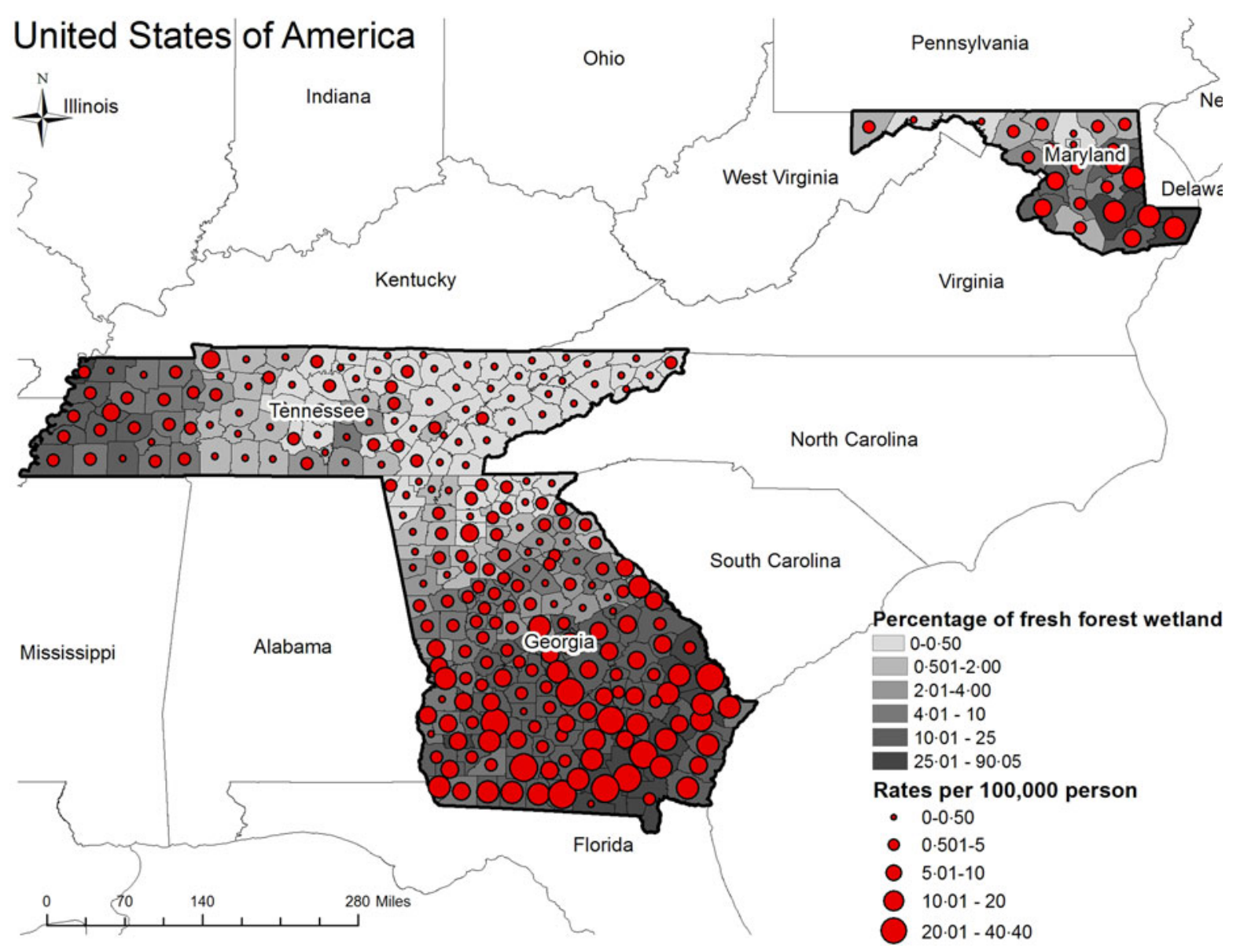

Fig. 1. Geographic distribution of Salmonella enterica serotype Javiana average incidence rates in Georgia, Maryland, and Tennessee, by county and percentage of fresh forested/scrub-shrub wetland present, 2005-2011. 
had the highest mean AvIR, 5.58 per 100000 persons, followed by Maryland (1.35), New Mexico (1.07), and Tennessee $(0 \cdot 80)$. County AvIR of serotype Javiana ranged from 0 to 40 per 100000 persons. In contrast, 3536 serotype Enteritidis cases were ascertained during our study period, and the mean county AvIRs were less variable among the four states. Mean AvIR of serotype Enteritidis was highest in Maryland (4.00) followed by Georgia (2.17), Tennessee (1.64), and New Mexico (1.43). County AvIR of serotype Enteritidis ranged from 0 to 19 per 100000 persons.

Figure 2 shows the correlation coefficients and 95\% confidence intervals for serotype Javiana county AvIR and wetland percent coverage for each wetland type by state. Fresh forested/scrub-shrub wetland was statistically significant in Georgia (0.70) and Maryland $(0 \cdot 66)$. The correlation between AvIR and the wetland percent coverage with fresh emergent wetland was also significant in Georgia (0.66) and Tennessee (0.42). We also found significant positive correlations in Georgia $(0 \cdot 29)$ and Tennessee $(0 \cdot 39)$ between AvIR and the wetland percent coverage with freshwater ponds wetlands. Other significant positive correlations included low positive correlations between AvIR and riverine wetlands in Georgia $(0 \cdot 34)$. In contrast, correlation estimates for serotype Enteritidis were not significant with wetland types in most sites except in Georgia, where a significant positive correlation was observed between AvIR with lakes (0.72), and a significant negative correlation between AvIR and fresh forested/scrub-shrub wetland (-0.27) (Fig. 2).

\section{DISCUSSION}

Studies of serotype Javiana have demonstrated regional differences in incidence rates and risk factors associated with environmental sources [4, 5, 12]. This analysis explored the relationship between wetland environments and incidence in order to assess whether regional differences in environmental habitats may be associated with observed variations in incidence. Specifically, in states with the highest AvIR, Georgia and Maryland, a strong positive correlation was found between serotype Javiana infection and wetland percent coverage with fresh forested/scrub-shrub. In Tennessee (lower AvIR) there was a positive correlation, but it was weaker. In contrast, serotype Enteritidis, typically associated with foodborne rather than environmental transmission [10], and was not associated with any wetland coverage except in Georgia. The results suggest that local differences in geology and hydrology may have an impact on the sources and frequency of transmission of serotype Javiana in the population - possibly related to animal reservoirs of the pathogen and to human activity in these environments.

Freshwater forested/scrub-shrub wetland is associated with wooded swamp habitats that may contribute to increased exposure to serotype Javiana compared with other wetland types. The wooded swamp habitats of the freshwater forested/scrub-shrub wetland support abundant forms of life, some of which may serve as reservoirs for serotype Javiana, or may contribute to the survival and environmental persistence of this serotype. For example, fresh forested/scrub-shrub wetland is an essential breeding ground for migratory birds and a critical habitat for juvenile fish and shellfish [13]. These habitats attract an array of wildlife, including many species of amphibians, which have been shown to be risk factors for serotype Javiana infection in case-control studies $[5,13]$. Living or spending time in these wetland areas may increase the probability of exposure to serotype Javiana. Furthermore, the unique characteristics of wetlands associated with increased serotype Javiana incidence may contribute to environmental survival and persistence of Salmonella; wetland habitats may support soil conditions and algae found to contribute to the environmental persistence of Salmonella [14, 15].

Fresh emergent wetlands coverage was significantly correlated with serotype Javiana incidence, but not to serotype Enteritidis, in Georgia, Maryland, and Tennessee. Another way wetland environments may lead to serotype Javiana exposure is through agriculture. During the dry season, farmers often use fresh emergent wetlands as farmland to grow produce. For example, in 2011, the 10 counties in Georgia with the most fresh emergent wetland coverage were the same counties that were ranked the top 10 in production value for produce [16]. The use of pesticides and other water contaminants during agricultural activities in these wetlands can disrupt the abilities of water quality enhancement or mitigating pesticide-runoff of wetlands [17]. Increase use of fertilizers also can result in increased concentration of nitrates and phosphates in associated farmlands which may foster bacterial growth, possibly serotype Javiana and its reservoirs [18, 19]. Studies have implicated irrigation water and the soil of tomato farms as environmental sources of serotype Javiana in produce [20], and have demonstrated that Salmonella can be 
Fresh Emergent Wetland
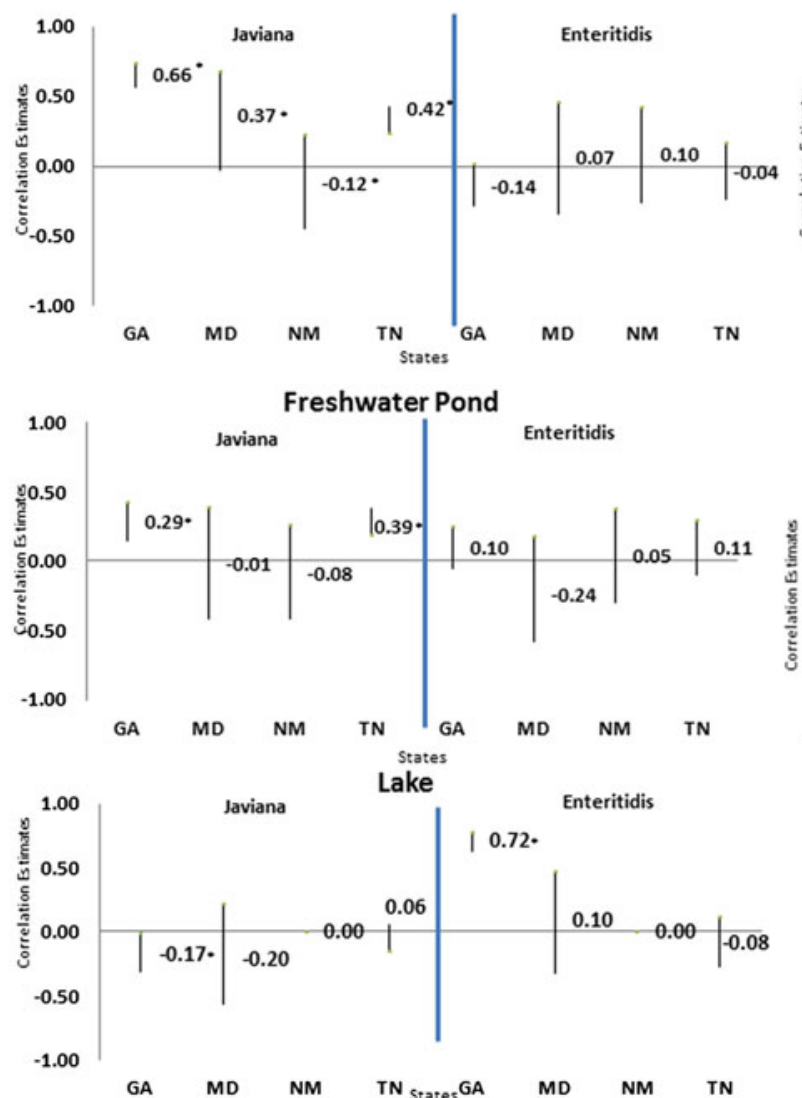

Freshwater Forested/Shrub Wetland
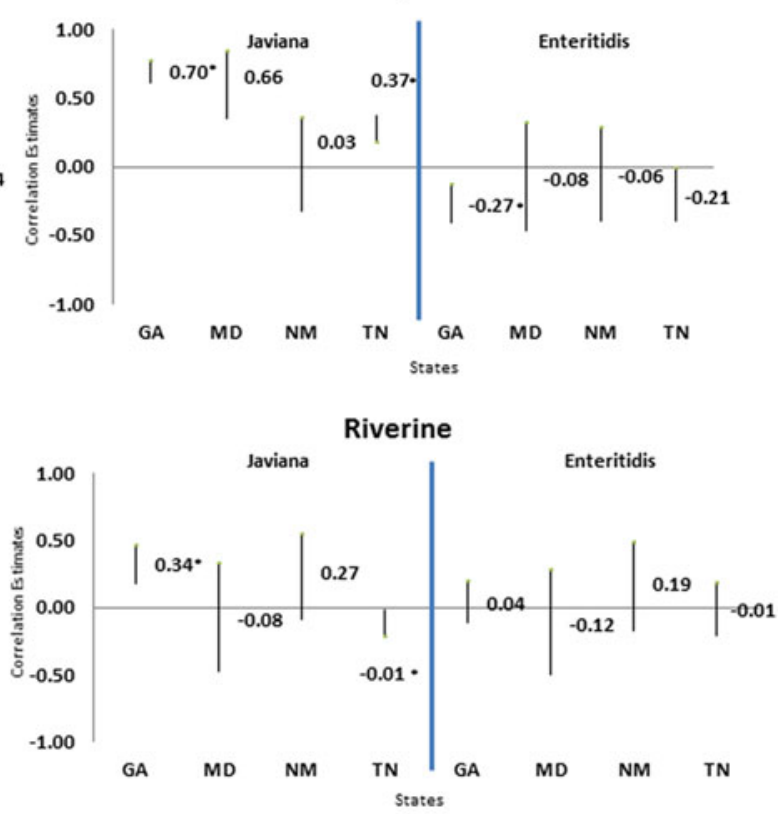

Fig. 2. Correlation coefficient and $95 \%$ confidence intervals of Salmonella enterica serotype Javiana and Salmonella enterica serotype Enteritidis in Georgia, Maryland, New Mexico, and Tennessee, by wetland type, 2005-2011. Abbreviations: GA, Georiga; MD, Maryland; NM, New Mexico; TN, Tenneesee. *Denotes statistical significance at $P<0 \cdot 05$.

transmitted inside plant blossoms or fruit [21]. The role of contaminated produce in the transmission of serotype Javiana is supported by a recent study that found $70 \%$ of serotype Javiana foodborne outbreaks were linked to plant-derived foods [3].

There was no correlation between serotype Javiana AvIR and wetland percent coverage in New Mexico counties. One reason for this may be that none of the wetland types exceeded a percent coverage > $0 \cdot 1 \%$ and associated habitat of the wetland types do not exist in New Mexico. Unlike Georgia, only 11\% of farms in New Mexico consisted of cropland and woodland; the majority of farms were classified as pasture [22]. With low percent coverage of any wetland type, our findings suggest that serotype Javiana infections in New Mexico may stem from different sources.

The analysis is limited to data aggregated at county level which did not take into account other factors that might influence observed correlations between serotype Javiana incidence rates and specific types of wetlands, such as speed and flow of the water, access to treated water, rainfall and temperature. Lack of a smaller geographic unit for analysis have a few limitations such as impossibility to include data on sociodemographic factors, socioeconomic status, education level, poverty level, and access to health care [23]. It is possible that these factors contributed to the associations identified in our study. For example, variations in incidence differ by race and ethnicity among infants and residents in counties with increased percentages of African American or Hispanic residents $[12,24,25]$. Conducting analyses at a smaller geographic unit (i.e. census tract) may help elucidate the interactions between these factors and the associations with wetlands found in this study. Likewise, analyses on a smaller geographic scale may clarify whether a specific wetland type is more influential as there might be spatial overlap between the different types of wetland since the study was conducted at the county level. Health care-seeking behaviors, access to health services, and other characteristics of the 
population in the surveillance area might affect the generalizability of the findings [26]. Another limitation is that incidence of serotype Javiana in Georgia is nearly five times higher than the incidence in the other 4 states included in our study, and correlations were observed for both serotype Javiana and serotype Enteritidis in Georgia.

\section{CONCLUSION}

The findings suggest that environmental habitats may support reservoirs or contribute to the persistence of serotype Javiana, and may frequently contribute to the transmission of infection compared with other Salmonella serotypes. Numerous significant positive correlations existed between the incidence of serotype Javiana infection and the presence of various wetland types in Georgia, Maryland, and Tennessee. Numbers of serotype Javiana infections have been continuously increasing since 2005 and the positive associations between the presence of specific wetlands and serotype Javiana in the Southeast suggest water and land use may introduce serotype Javiana into associated farmlands. Furthermore, the crops grown in wetland environments could play an important role in human infections. Spatial analysis can be a valuable tool for assessing the possible relationship between Salmonella and environmental sources. This ecological study highlights the importance of incorporating geospatial data in the examination of demographic and socioeconomic factors. Future studies to better understand these findings and develop targeted education and prevention tools are important.

\section{ACKNOWLEDGEMENTS}

The authors would like to thank CDC Emerging Infections Program, FoodNet sites, U.S. Department of Agriculture's Food Safety and Inspection Service, U.S. Food and Drug Administration, and US Fish and Wildlife Services for the National Wetland Inventory.

\section{DECLARATION OF INTEREST}

None.

\section{REFERENCES}

1. Scallan E et al. Foodborne illness acquired in the United States-major pathogens. Emerging Infectious Diseases 2011; 17: 7-15.
2. Centers for Disease Control. National Salmonella Surveillance Annual Data Summary, 2011. Atlanta, Georgia: US Department of Health and Human Services, Centers for Disease Control and Prevention, 2013.

3. Jackson BR et al. Outbreak-associated Salmonella enterica serotypes and food commodities, United States, 1998-2008. Emerging Infectious Diseases 2013; 19: 1239-1244.

4. Mermin $\mathbf{J}$ et al. Emerging infections program FoodNet Working Group. Reptiles, amphibians, and human Salmonella infection: a population-based, casecontrol study. Clinical Infectious Diseases 2004; 38: S253-S261.

5. Srikantiah $\mathbf{P}$ et al. Salmonella enterica serotype Javiana infections associated with amphibian contact, Mississippi, 2001. Epidemiology and Infection 2004; 132: 273-281.

6. Baudart $\mathbf{J}$ et al. Diversity of Salmonella strains isolated from the aquatic environment as determined by serotyping and amplification of the ribosomal DNA spacer regions. Applied and Environmental Microbiology 2000; 66: $1544-1552$

7. ESRI. ArcGIS Desktop: Release 10. Redlands, CA: Environmental Systems Research Institute, 2011.

8. Cowardin LM, et al. Classification of Wetlands and Deepwater Habitats of the United States. Washington, DC; Jamestown, ND: U.S. Department of the Interior, Fish and Wildlife Service, 1979.

9. Zar JH. Spearman rank correlation. Encyclopedia of Biostatistics 2005; 7: 1-5.

10. Voetsch AC et al. Analysis of the FoodNet case-control study of sporadic Salmonella serotype enteritidis infections using persons infected with other Salmonella serotypes as the comparison group. Epidemiology and Infection 2009; 137: 408-416.

11. SAS. Base $S A S^{\circledR} 9.3$ Procedures Guide. Cary, NC: SAS Institute Inc., 2011.

12. Chang $\mathbf{M}$ et al. An ecological analysis of sociodemographic factors associated with the incidence of salmonellosis, shigellosis, and E. coli O157:H7 infections in US counties. Epidemiology and Infection 2009; 137: $810-820$.

13. Shellenbarger GG et al. Fecal indicator bacteria and Salmonella in ponds managed as bird habitat, San Francisco Bay, California, USA. Water Res 2008; 42: 2921-2930.

14. Byappanahalli MN et al. Seasonal stability of Cladophora-associated Salmonella in Lake Michigan watersheds. Water Research 2009; 43: 806-814.

15. Erickson MC et al. Examination of factors for use as potential predictors of human enteric pathogen survival in soil. Journal Applied Microbiology 2013; 116: 335-349.

16. Development C.f.A.a.E. 2012 Georgia Farm Gate Value Report. Volume AR-13-01. Athens, GA: The University of Georgia, 2013.

17. Moore M et al. Constructed wetlands for mitigation of atrazine-associated agricultural runoff. Environment Pollution 2000; 110: 393-399.

18. Lundberg $\mathbf{J}$ et al. Nitrate, bacteria and human health. Nature Reviews Microbiology 2004; 2, 593-602. 
19. DebRoy S et al. Isolation of nitrate and phosphate removing bacteria from various environmental sites. OnLine Journal of Biological Sciences 2012; 12: 62-71.

20. Micallef SA et al. Occurrence and antibiotic resistance of multiple Salmonella serotypes recovered from water, sediment and soil on mid-Atlantic tomato farms. Environmental Research 2012; 114: 31-39.

21. Guo X et al. Survival of Salmonella on and in tomato plants from the time of inoculation at flowering and early stages of fruit development through fruit ripening. Applied and Environmental Microbiology 2001; 67: 4760-4764.

22. NASS. In: Agriculture USDo, ed. Agricultural Statistics 2009. Chapter 7, pp. 6-10. Washington, DC: U.S. Government Printing Office, 2009.

23. Cromley EK, McLafferty SL. GIS and Public Health, 2nd edn. New York, NY: Guilford Press, 2012.
24. Cheng LH, et al. Epidemiology of infant Salmonellosis in the United States, 1996-2008: a foodborne diseases active surveillance network study. Journal Pediatric Infectious Diseases 2013; 2: 232-239.

25. Vugia DJ et al. Emerging infections program FoodNet Working Group. Invasive Salmonella infections in the United States, FoodNet, 1996-1999: incidence, serotype distribution, and outcome. Clinical Infectious Disease 2004; 38: S149-S156.

26. Huang JY et al. Infection with pathogens transmitted commonly through food and the effect of increasing use of culture-independent diagnostic tests on surveillance - foodborne diseases active surveillance network, 10 U.S. sites, 2012-2015. MMWR Morbidity and Mortality Weekly Report 2016; 65: 368-371. 